\title{
Editorial: A Cross-Section of the Current Research on High-Temperature Superconductivity
}

Discovering new high-temperature superconductors and understanding their physics have been a quest in condensed matter physics. Fortuitously, a collection of three papers on three different classes of high-temperature superconductors of current interest has come together as a part of the inaugural issue of Physical Review X. The paper by a group of theorists from Purdue and Princeton Universities as well as the Institute of Physics of the Chinese Academy of Sciences (Fang et al.) investigates microscopic mechanisms responsible for electron pairing in iron-based superconductors; the experimental paper by a Chinese group at Fudan University in Shanghai in collaboration with a group at the Polish Academy of Sciences (Dong et al.) reports new experimental data shedding light on a recently discovered heavy-fermion superconductor; and finally, the theoretical paper by Yildirim and Ku from Brookhaven National Laboratory presents an intriguing theory for explaining the origin and puzzling behavior of the superconducting gap in underdoped cuprates. We are publishing these three papers together today and leading the publication with a brief commentary on each written by PRX Editorial Board members Hong Ding and Barbara Jones, and an invited guest commentator, Assa Auerbach. It is our hope that this effort puts the three papers on a broader canvas and will be an added value to you as a reader, enhancing your understanding and appreciation of these publications. We will continue to make such efforts when similar opportunities present themselves in the future.

The Editors

Physical Review X

\section{Determining Microscopic Pairing Mechanisms by Identifying Pairing Symmetry in Iron-Based Superconductors}

Hong Ding, Institute of Physics, The Chinese Academy of Sciences, PO Box 603, Beijing 100190, CHINA

A Commentary on:

Robustness of $s$-Wave Pairing in Electron-Overdoped $A_{1-y} \mathrm{Fe}_{2-x} \mathrm{Se}_{2}(A=\mathrm{K}, \mathrm{Cs})$

Chen Fang, Yang-Le Wu, Ronny Thomale, B. Andrei Bernevig, and Jiangping Hu

Phys. Rev. X 1, 011009 (2011)

Pairing symmetry is one of the most fundamental characters of superconducting states. Different microscopic pairing mechanisms can result in different pairing symmetries. In the case of iron-based superconductors, weak-coupling approaches based on pairing mediated by spin excitations predict a $d$-wave pairing symmetry for heavily electron-doped chalcogenides $A_{1-y} \mathrm{Fe}_{2-x} \mathrm{Se}_{2}$ and an $s^{ \pm}$wave for optimally doped iron pnictides such as $\mathrm{BaFe}_{2-x} \mathrm{Co}_{x} \mathrm{As}_{2}$, and other approaches assuming orbital-fluctuation mediated pairing suggest $s^{++}$-wave pairing symmetry for both materials. In their newly published paper in Physical Review X, Fang et al. show that the pairing symmetry should remain a robust $s$-wave pairing symmetry for both materials if pairing is mainly caused by local next-nearest-neighbor antiferromagnetic exchange couplings. The $s$-wave symmetry is manifested as $s^{++}$for the chalcogenides and $s^{ \pm}$for the pnictides. Therefore, this work suggests that determining pairing symmetry will provide important information regarding microscopic pairing mechanisms in iron-based superconductors.

Published by the American Physical Society under the terms of the Creative Commons Attribution 3.0 License. Further distribution of this work must maintain attribution to the author(s) and the published article's title, journal citation, and DOI. 


\section{Heavy-Fermion Superconductivity}

Barbara Jones, IBM Almaden Research Center, 650 Harry Road, San Jose, CA 95120-6099, USA

A Commentary on:

Field-Induced Quantum Critical Point and Nodal Superconductivity in the Heavy-Fermion Superconductor $\mathrm{Ce}_{2} \mathrm{PdIn}_{8}$

J. K. Dong, H. Zhang, X. Qiu, B. Y. Pan, Y. F. Dai, T. Y. Guan, S. Y. Zhou, D. Gnida, D. Kaczorowski, and S. Y. Li

Phys. Rev. X 1, 011010 (2011)

Heavy-fermion superconductors are characterized by an often complex competition between superconductivity and antiferromagnetism, with non-Fermi-liquid phases also a feature of the normal states in proximity to a quantum critical point. A positive identification of the superconducting order parameter and a determination of the microscopic origin of the superconductivity remain to be some of the outstanding challenges. Heavy-fermion superconductors can have $d$ and even $f$ symmetry, as well as exotic variants on these. In the paper by Dong et al. on $\mathrm{Ce}_{2} \mathrm{PdIn}_{8}$ in this three-paper series, reported observations include nodal superconductivity and a magnetic-field-induced quantum critical point, suggesting that, as in the related compound $\mathrm{CeCoIn}_{5}$, a Fulde-Ferrell-Larkin-Ovchinnikov (FFLO) state or other unconventional superconducting states may exist. Determining the magnetic component to the quantum critical point via, for example, neutron scattering will be important future work suggested by this research.

\section{Superconductivity in the Cuprates}

Assa Auerbach, Department of Physics, Technion - Israel Institute of Technology, 32000 Haifa, ISRAEL

A Commentary on:

Kinetics-Driven Superconducting Gap in Underdoped Cuprate Superconductors within the Strong-Coupling Limit

Yucel Yildirim and Wei Ku

Phys. Rev. X 1, 011011 (2011)

Underdoped cuprate superconductors exhibit unusual electronic spectral properties. Above $T_{c}$, a "pseudogap" is found in the antinodal directions in the Brillouin zone. Below $T_{c}$, another gap opens near the nodal regions that seems to exhibit different temperature and doping dependence. This apparent breakdown of the Fermi-liquid theory for the normal state, and of the Bardeen-Cooper-Schrieffer theory for the superconducting state, has puzzled theorists for some time, and spurred intense experimental interest in the pseudogap phase. In the intriguing paper published in Physical Review X, Yildirim and Ku have analyzed the "two gaps" phenomenology using a simple boson-fermion Hamiltonian. The bosons are short-ranged $d$-wave hole pairs, and the fermions are unpaired holes. The bosons can dissociate (an Andreev process) into two fermions with relative $d$-wave symmetry. Below $T_{c}$, the gap near the $d$-wave nodes is proportional to the bosonic superconducting order parameter. The nodal gap can therefore be uncorrelated to the fermionic excitations near the antinodes. The attractiveness of such a two-fluid description (which has also been proposed before, but received little attention from the community) is that it may provide an alternate route to "non-Fermi-liquid" transport and thermodynamics such as those observed in the enigmatic pseudogap phase. 
About the three commentary authors:

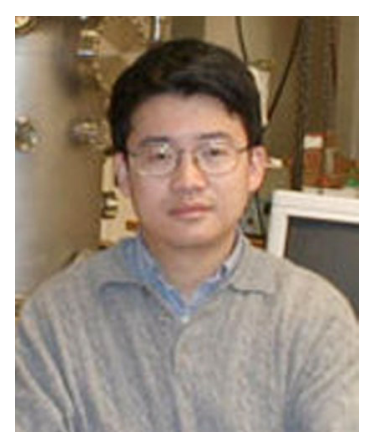

Hong Ding is Distinguished Professor and Chief Scientist in the Beijing National Laboratory for Condensed Matter Physics at the Institute of Physics of the Chinese Academy of Sciences. His group carries out cutting-edge experimental research in high- $T_{c}$ superconductors.

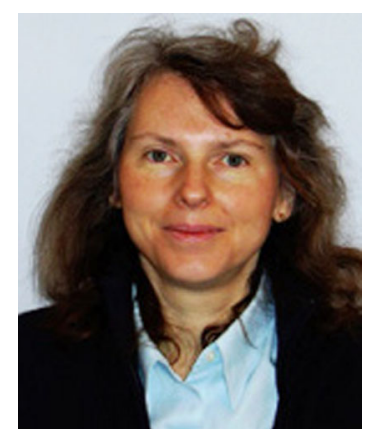

Barbara Jones is a research staff member at IBM Almaden Research Center, where she leads the theoretical and computational physics group. Her research concentrates on systems of strongly correlated electrons and on quantum magnetism.

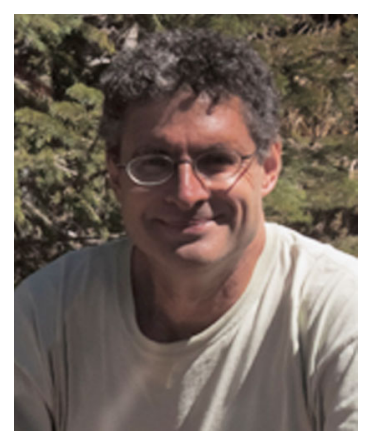

Assa Auerbach is the Sydney and Elizabeth Corob Professor of Exact Sciences at the Physics Department of Technion - Israel Institute of Technology. His research spans a broad range of topical areas in condensed matter physics, including strongly correlated electron and boson systems, high-temperature superconductivity, and quantum magnetism.

Published 15 September 2011

DOI: 10.1103/PhysRevX.1.010001

PACS numbers: 01.30.Ww 